\title{
SAFETY AND HEALTH SITE INSPECTIONS FOR ON-FIELD RISK ANALYSIS AND TRAINING
}

\author{
MARCO L. TRANI ${ }^{1}$, LUCA BERETTA ${ }^{2}$, GIADA NICOLINI ${ }^{3}$, and MANUELE CASSANO ${ }^{1}$ \\ ${ }^{1}$ Politecnico di Milano, A.B.C. Dept, Milan, Italy \\ ${ }^{2}$ CPT Milano, Assago, Italy \\ ${ }^{3}$ Research Consultant, Lesmo (MB), Italy
}

\begin{abstract}
The field of construction is always affected by a large number of accidents at work that have many different causes and responsible. Therefore, it is of utmost importance to focus on all these issues, in order to reduce all risk factors that can undermine individuals' safety on building sites. The objective of the research is then the development of a method for quick on site analysis of all critical issues that can create accidents and identification of the related causes in order to directly provide a correct and focused training identified as the best method to act on the causes to reduce accidents. The research was carried on during-construction of the Universal Exhibition of Milan - Expo 2015 - that counted almost 70 contemporary construction sites. To reach the goals further research steps has been followed and in particular: (i) inspections on building sites through all the Expo area; (ii) analysis of the main identified problems; (iii) development of a methodology to quickly identify the cause of problems; (iv) validation of the method through back office analysis of site documents; (v) correct on-site training according to found problem. During the whole construction site, the improvements in criticalities solving have been visible thanks to the focused training. The developed method, carried on in a high-risk environment, is applicable in any other building sites and environment as independent from the boundary conditions of the place.
\end{abstract}

Keywords: Construction critical issues, Site inspections, Training.

\section{INTRODUCTION}

Nowadays the construction appears to be in Italy one of the fields with the largest number of accidents at work (INAIL 2015). Our experience told us that training courses are the main vehicle to develop a proper safety skill, but only if deal directly with potentially dangerous situations. Today the two main standards for health and safety training in Italy are: the Legislative Decree no. 81/2008 and the State-Regions Agreement of 2011. In particular, the latter defines duration, contents and methods of training based on the risk considered for the specific activity: low, medium, high. However, we think that it is also essential to calibrate topics and duration of training courses in order to be perfectly aligned with the critical issues present in the construction site and that workers have to face every day. Demirkesen and Arditi (2015) think that effectiveness of safety training practices is very important and analyzed if this consciousness is present in professionals' perceptions in order to understand how training can be improved. Choudhry (2014) worked in particular on critical behavior of workers in order to improve safety performances. Behavior-based Safety (BBS) was considered also by Zhang and 
Fang (2013) that try to introduce this practice into management routine. Finally Yang et al. (2012) recognized training as one of the requirements for accident identification and implemented a real-time identification system. It is visible then how on site direct supervision and training can improve site safety by acting directly on accident causes determined on field. The presented research aims in particular to categorize the main accident causes in order to find the best solution in terms of workers training. Then site inspections permitted to develop a method for a direct evaluation of the cause and the responsible of the problem. Finally tests and office analysis on the documentation permitted to validate the developed method in order to be used consistently in future construction site. The output of the research is an "on the job" training focused on the causes of the faced problems. These research project arise from a partnership between bilateral bodies (CPT Milano) and INAIL (The National Insurance Institute Against Accidents at Work) of the Lombardy region and has been developed in teamwork with Politecnico di Milano.

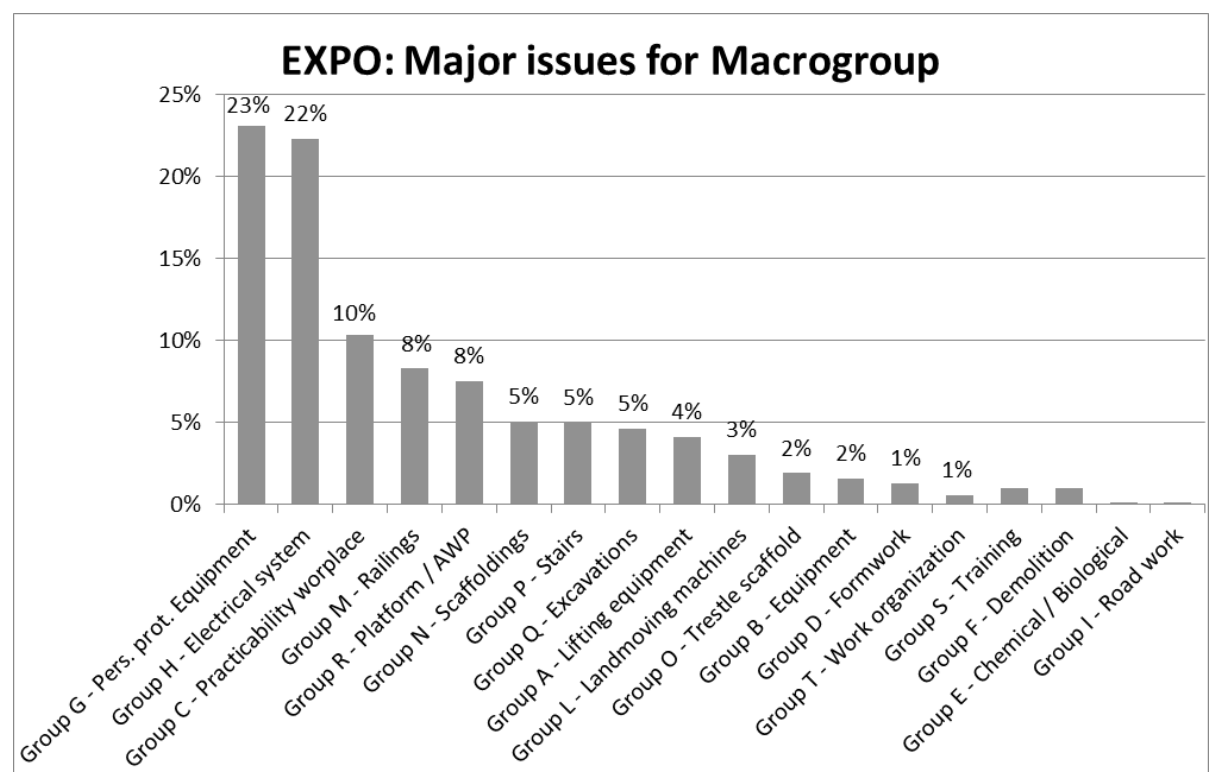

Figure 1. Major issues for Macro group checking during inspections.

\section{MONITORING ANALYSIS}

The experimental analyzes have been conducted by the task group of Politecnico di Milano on the basis of data obtained during the inspections carried out in the sites in Milan - EXPO 2015 by the CPT technicians. The main work of the CPT was to do inspections on sites aimed to a continuous assistance on safety matters. This help was offered to construction companies and to workers all over the Expo site, and was directed to the identification of any critical situation. Statistics on visits carried out by CPT engineers and trainers are the following:

- Number of sites monitored: 69

- Number of companies involved: 799

- Number of visits carried out: 683

- Number of employees trained: 1314

- Number of total of problems found: 4829 


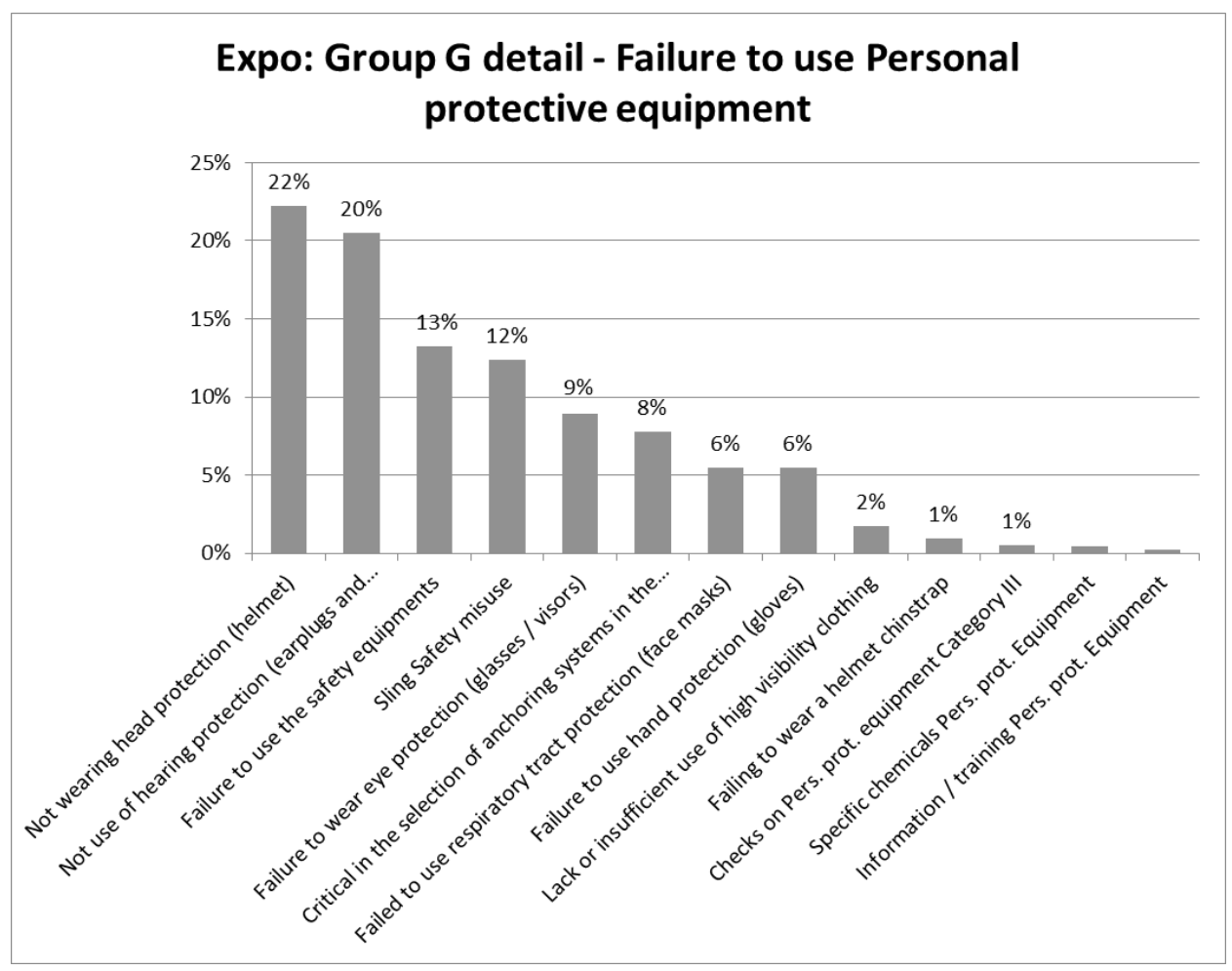

Figure 2. Macro-group details relating to personal protective equipment.

During inspections, the technicians analyzed and controlled all the works in progress, checking the proper performances compared to existing regulatory dictate, thus offering advices to firms' technicians or training support directly to workers. Once completed the monitoring activities, we proceed with the preparation of two documents for the check of construction activities, the first to be compiled by the technician and the second, based on the first one, to be compiled by the trainer. These verification cards were drawn up for each individual work in action (for example: mounting scaffolding, excavations, etc.), for each site and for each specific company. The technical report, developed from extensive experience of established systems of $\mathrm{CPT}$, takes into account all the main activities that are potentially carried out in the construction site and shows, for each of them, all potential sources of risk. This creates the basic matrix consisting of two levels. The first is related to a specific subject area while the second is related to a specific critical issue. Table 1 shows the 18 captions that includes about 180 criticalities.

Table 1. List of identified subject areas.

\begin{tabular}{|l|l|l|}
\hline "A" - Lifting equipment & "G"- Pers. prot. equipment & "O" - Trestle scaffold \\
\hline "B" - Equipment & "H" - Electrical system & "P" - Ladder \\
\hline "C" - Practicability workplace & "I" - Road works & "Q" - Excavations \\
\hline "D" - Formwork & "L"- Land moving machines & "R" - Platform / AWP \\
\hline "E" - Chemical / Biological & "M"- Railings & "S" - Training \\
\hline "F"- Demolition Total / Partial & "N"- Scaffolding & "T" - Organization of work \\
\hline
\end{tabular}


From these data it was possible to perform an analysis which allowed to have a global and detailed view of the whole criticalities. It has been conducted a first level of analysis on major nonconformity found for broad groups (see graphic in Figure 1 for the major issues found). It was then possible to deepen the analysis of non-compliances detected down to a second level of detail. Graphic in figure 2 shows the details of the personal protective equipment macro-group.

\section{EVALUATION METHOD}

Starting from the outcome of technical assistance on site, we tried to find unique ways to bring works non-conformities at three different conditions specified in these areas:

- Behavioral area, where the problem is directly due to the worker's working method;

- Organization corporate area, where the problem is due to deficient equipment provision;

- Area related to the organization of the workplace, where the problem is due to a wrong control of individual processes and work contexts in which they occurred.

A specific method has been set up for on-field evaluation of the non-conformities and the related areas. It is mainly based on the analysis of the time required to resolve problems encountered. In the pipeline it was assumed the amenability of the same problem to the three areas described above. Three resolution times were considered and at the three levels were associated different assignments, related to the causes of the critical issue:

- $\quad \mathrm{ST}=$ Short time: $5 / 10 \mathrm{~min}=$ Behavioral problems $(\mathrm{L})$, whose almost immediate solution can be fielded directly by the worker with tools that they already have

- $\quad$ MT = Medium time: medium time due to the duration of the monitoring carried out = Labor Organization $(\mathrm{O})$, the resolution of which potentially requires the intervention of multiple resources, human and technical, which can therefore be put in place by those who have functional power directly on site

- $\mathrm{LT}=$ Long time: Attributable to cases in which the resolution is greater than the monitoring activity $=$ Organization $(\mathrm{M})$, the solution of which necessarily requires the intervention of company top management.

The method, used to be able to support the above-mentioned hypothesis, takes place starting from the monitoring carried out in the construction site followed by back office analysis. The starting point for this investigation is developed considering primarily the inspections carried out by the technicians and CPT trainers in the various construction sites with the detection of any critical issues for each machining process. After reporting the criticality to the worker and/or to the person in charge all timing resolution of every problem were monitored. The analysis consists to check how quickly the critical issues was resolved, compared to the same period of monitoring, distinguishing them in the three times above. This method is established with the aim of being able to carry out the feedback on the cause of the criticality directly on site, quickly, without losing days behind the cards.

\section{VALIDATION}

In order to validate this method, we proceed with a deeper back office analysis. In fact we must be sure that the time consumed for a criticality resolution can be associated to one of the above mentioned areas in order to be certain where intervene. 


\section{IMPROPER USE OF MOBILE ELEVATING WORK PLATFORMS (AWP)}
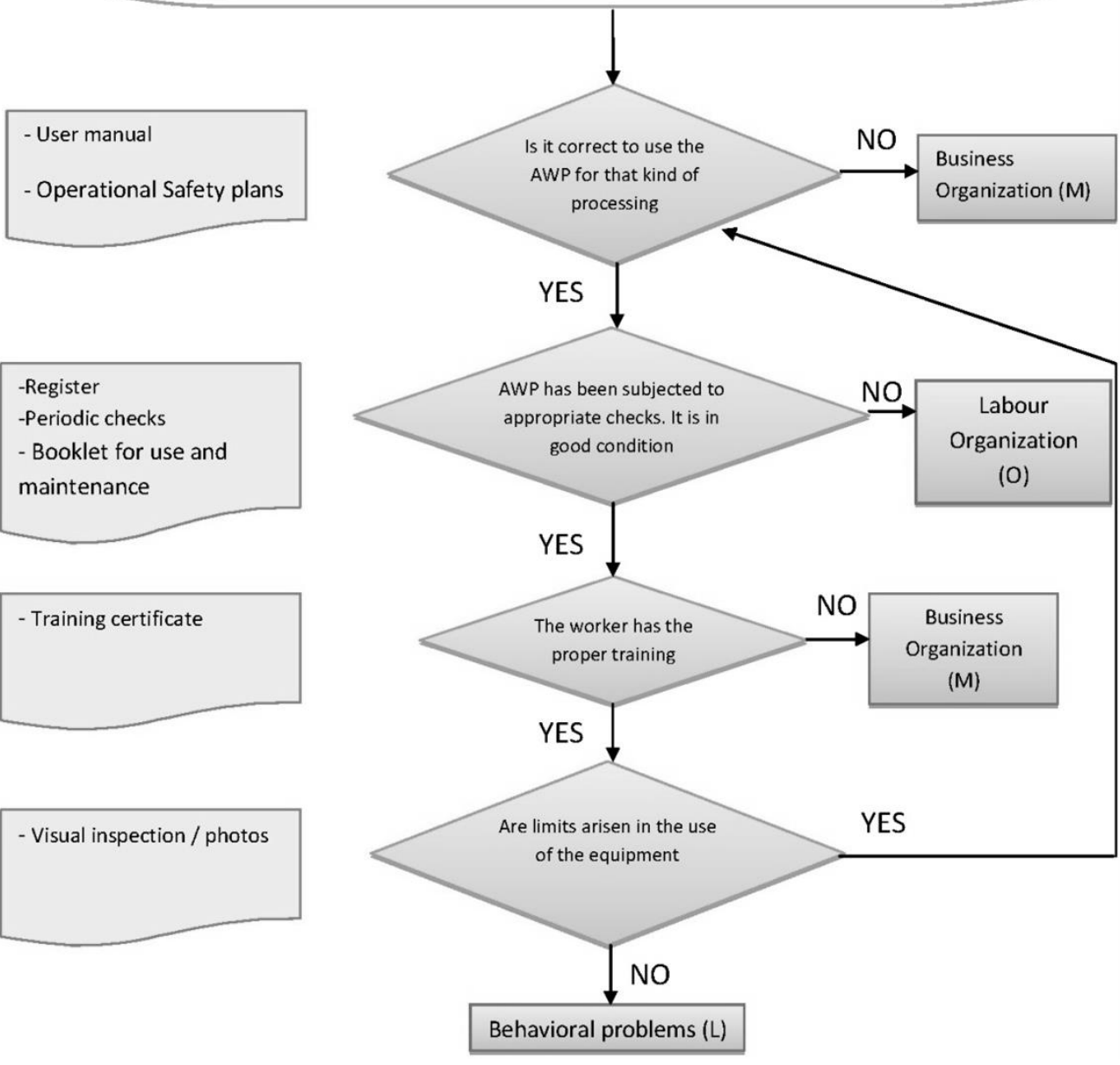

Figure 3. Example of flow chart: improper use of AWP.

This analysis involves, in the first instance, the use and study of documents provided by the companies (Operational Safety plans, Safety procedures, Training certificates, etc.) and secondly the use of flow charts made for this purpose for each single Macro-group. Each Macro-group has its own flow chart to figure out the cause of the criticality. The flow charts concern each examined item during the technical assistance activities. For each operation described in the diagrams, we have identified different outputs, so that we could arrive to identify the subjects that made the non-compliance. Figure 3 shows one of the twelve flow charts created for this purpose.

Applying this methodology, over a sample of issues found in the construction sites, we obtained an estimated percentage useful to determine the capabilities of the instrument adopted. Figure 4 shows the summary graphs of such analysis. The $96 \%$ of the solved situations in the short term relates with the behavioral sphere of the individual worker. It remains a $4 \%$ due to the necessary intervention of a subject with organizational power than the other assigned work. As in the previous case, you may notice that there is no automatic allocation of a responsibility given based on the time taken to solve a problem. Each situation should be carefully considered in order to understand from where derives the error returned. In the case shown the mismatch is 
even more striking: $30 \%$ of the situations resolved in a high amount of time, by default attributed to the apex of the company, is actually due to an intervention required to responsible business.

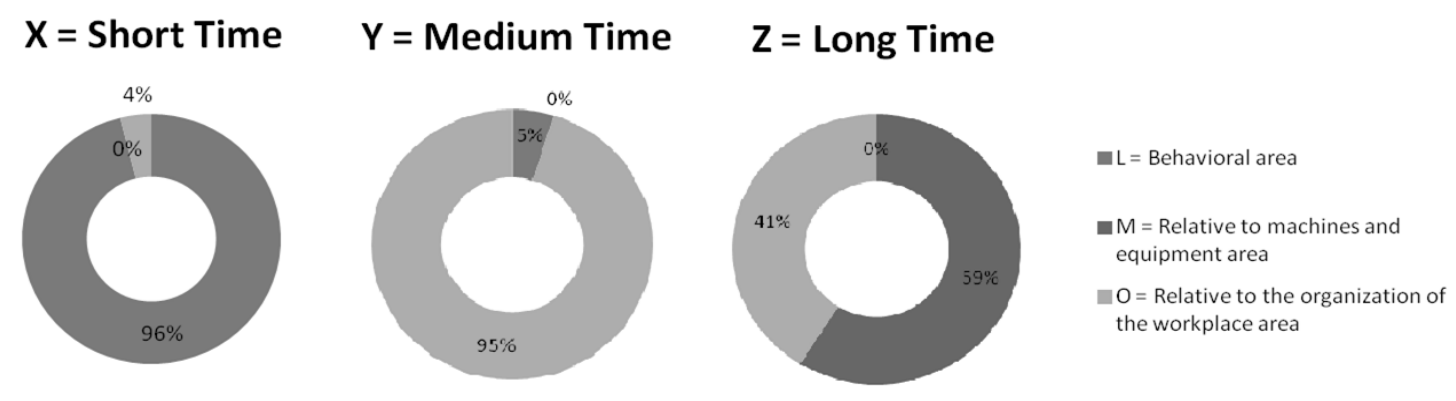

Figure 4. Percentage about situation solved in Short / Medium / Long time.

It's possible to underline that the adopted instrument responded positively to the objective.

\section{CONCLUSIONS}

In conclusion, this research has allowed us to create a model to neatly categorize, and then rework in statistical form, the data collected during the monitoring conducted in the Expo sites, aimed at business support and training for workers. This model, referred to the problems encountered, allowed to obtain evidence of risk situations identified above and, to bring out the causes that generated them. The knowledge of the causes allowed to direct interlocutors to the best methods of troubleshooting, also suggesting actions to be taken to prevent their recurrence in time. It has thus developed a first proposal for the basic training course for workers in the field of health and safety, able to optimize the timing and subject matter, based on the obtained statistical data and the comparison with the teachers of the training courses themselves.

Furthermore, this research has allowed us to confirm that the training provided in the 2011 State-Regions must be developed according to the task, and integrated with the introduction of greater specificity in terms of risk classes and type of work. This model, made from data obtained in a high risk environment, can be used in any other type of construction site and can be implemented in national and international level. Also well suitable for application in any type of environment as independent of the boundary conditions of the place, ensuring that it can be seen as an expendable standard course for all companies in the construction sector.

\section{References}

Choudhry, R. M., Behavior-based safety on construction sites: A case study, Accident Analysis \& Prevention, Volume 70, 14-23, 2014.

Demirkesen, S., Arditi, D., Construction safety personnel's perceptions of safety training practices, International Journal of Project Management, 33(5), 1160-1169, 2015.

Italian Law 81/2008 on workers' health and safety and its following modifications (2008).

Italian State-Regions Agreement about safety training (2011).

INAIL (The National Insurance Institute Against Accidents at Work). Database of accidents at work (2015).

Yang, H., Chew, D.A.S., Wu, W., Zhou, Z., Li, Q., Design and implementation of an identification system in construction site safety for proactive accident prevention, Accident Analysis \& Prevention, Volume 48, 193-203, 2012.

Zhang, M., Fang, D., A continuous Behavior-Based Safety strategy for persistent safety improvement in construction industry, Automation in Construction, Volume 34, 101-107, 2013. 